\title{
Duration of Humoral and Cellular Immunity 8 Years After Administration of Reduced Doses of the 17DD-Yellow Fever Vaccine
}

OPEN ACCESS

Edited by:

Karl Ljungberg,

Karolinska Institute (KI), Sweden

Reviewed by:

Roger E. Thomas,

University of Calgary, Canada

Jorma Hinkula,

Linköping University, Sweden

Anna Helena Roukens,

Leiden University Medical

Center, Netherlands

${ }^{*}$ Correspondence:

Ana Carolina Campi-Azevedo campiazevedo@gmail.com

Olindo Assis Martins-Filho

oassismartins@gmail.com

Specialty section:

This article was submitted to Vaccines and Molecular Therapeutics,

a section of the journal

Frontiers in Immunology

Received: 19 March 2019

Accepted: 13 May 2019

Published: 21 June 2019

Citation:

Costa-Rocha IAd, Campi-Azevedo

$A C$, Peruhype-Magalhães $V$,

Coelho-dos-Reis JG, Fradico JRB,

Souza-Lopes T, Reis $L R$, Freire LC,

Costa-Pereira C, Mambrini JVdM,

Maia MdLdS, Lima SMBd, Noronha

$T G d$, Xavier JR, Camacho LAB,

Albuquerque EMd, Farias RHG

Castro TdMd, Homma A, Romano APM, Domingues CM, Martins RdM,

Teixeira-Carvalho $A$ and Martins-Filho

OA (2019) Duration of Humoral and

Cellular Immunity 8 Years After

Administration of Reduced Doses of

the 17DD-Yellow Fever Vaccine.

Front. Immunol. 10:1211.

doi: 10.3389/fimmu.2019.01211
Ismael Artur da Costa-Rocha ${ }^{1}$, Ana Carolina Campi-Azevedo ${ }^{1 *}$, Vanessa Peruhype-Magalhães ${ }^{1}$, Jordana Grazziela Coelho-dos-Reis ${ }^{1,2}$, Jordana Rodrigues Barbosa Fradico ${ }^{1}$, Thalles Souza-Lopes ${ }^{1}$, Laise Rodrigues Reis ${ }^{1}$, Larissa Chaves Freire ${ }^{1}$, Christiane Costa-Pereira ${ }^{1}$, Juliana Vaz de Melo Mambrini ${ }^{3}$, Maria de Lourdes de Sousa Maia ${ }^{4}$, Sheila Maria Barbosa de Lima ${ }^{5}$, Tatiana Guimarães de Noronha ${ }^{4}$, Janaina Reis Xavier ${ }^{4}$, Luiz Antonio Bastos Camacho ${ }^{6}$, Elizabeth Maciel de Albuquerque ${ }^{4}$, Roberto Henrique Guedes Farias ${ }^{7}$, Thalita da Matta de Castro ${ }^{4}$, Akira Homma ${ }^{4}$, Alessandro Pecego Martins Romano ${ }^{8}$, Carla Magda Domingues ${ }^{8}$, Reinaldo de Menezes Martins ${ }^{4}$, Andréa Teixeira-Carvalho ${ }^{1}$ and Olindo Assis Martins-Filho ${ }^{1 *}$

' Grupo Integrado de Pesquisas em Biomarcadores, Instituto René Rachou, Fundação Oswaldo Cruz - FIOCRUZ-Minas, Belo Horizonte, Brazil, ${ }^{2}$ Laboratório de Virologia Básica e Aplicada, Departamento de Microbiologia, Instituto de Ciências Biológicas, Universidade Federal de Minas Gerais, Belo Horizonte, Brazil, ${ }^{3}$ Núcleo de Estudos em Saúde Pública e Envelhecimento, Instituto René Rachou, Fundação Oswaldo Cruz - FIOCRUZ-Minas, Belo Horizonte, Brazil, ${ }^{4}$ Assessoria Clínica, Instituto de Tecnologia em Imunobiológicos Bio-Manguinhos - FIOCRUZ, Rio de Janeiro, Brazil, ${ }^{5}$ Laboratório de Tecnologia Virológica, Instituto de Tecnologia em Imunobiológicos Bio-Manguinhos - FIOCRUZ, Rio de Janeiro, Brazil, ${ }^{6}$ Departamento de Epidemiologia e Métodos Quantitativos em Saúde - Escola Nacional de Saúde Pública - FIOCRUZ, Rio de Janeiro, Brazil, ${ }^{7}$ Instituto de Biologia do Exército, Rio de Janeiro, Brazil, ${ }^{8}$ Departamento de Vigilância das Doenças Transmissíveis, Secretaria de Vigilância em Saúde, Ministério da Saúde, Brasilia, Brazil

The present study aims to determine whether 17DD-YF-specific humoral and cellular immunological memory is maintained 8-years after primary vaccination with subdoses (10,447IU;3,013IU;587IU;158IU;31IU). For this purpose, this follow-up study was carried out in a subset of volunteers $(n=98)$ originally enrolled in the dose-response study in 2009 and 46 non-vaccinated controls. Our results demonstrated that vaccinees, who had seroconverted following primary vaccination and had not been revaccinated, present similar neutralizing antibodies levels and YF-specific cellular memory, particularly CMCD4 and EMCD8 as compared to the reference full dose $(27,476 \mathrm{IU})$. Although, PRNT seropositivity rates were similar across subgroups $(94,82,83,94,80$, and $91 \%$, correspondingly), only doses above 587IU elicited similar iterative proportion of seropositivity rates, calculated as a progressive decrease on seropositivity rates along time (89, 80, 80, and 91\%, respectively) as compared to 158IU and $31 \mathrm{IU}$ (68 and $46 \%$, respectively). Noteworthy were the strong positive correlations ("EMCD4,EMCD8" and "TNFCD8,IFNCD8") observed in most subdoses, except for 31IU. Major similarities underscored the preserved antibody titers and the outstanding levels of EMCD8, relevant correlates of protection for YF-specific immunity. These findings provide evidences to support the regular use of dose sparing strategy for YF vaccine in adults.

Keywords: Yellow Fever, 17DD vaccine, subdoses, neutralizing antibodies, cellular memory 


\section{INTRODUCTION}

Yellow fever (YF) is a severe acute febrile infectious disease, transmitted by mosquitoes infected with a Flavivirus RNA that occurs in Latin America and Africa. The disease is more frequent in non-immunized travelers entering into YF-endemic areas and non-vaccinated young males living in YF-endemic countries due to incursions in sylvatic areas of YF viral circulation $(1,2)$.

YF represents a substantial risk for non-immunized travelers entering YF-endemic areas and especially to residents of YFendemic countries $(1,2)$. Because there is no effective treatment for YF, the prevention by immunization is critical to reduce the risks of YF infection. The YF vaccination has been considered the most relevant and effective prophylactic measure, inducing protective immunity within $10-30$ days in $\sim 95-99 \%$ of primary adult vaccinees $(1,3,4)$.

The current outbreaks of YF in Brazil and Africa (5-10) have increased the demand for YF vaccine with consequent depletion of international stockpile. In response to this scenario, the World Health Organization (WHO) has recommended the use of fractional dose strategy to prevent the YF spread $(11,12)$. The fractional dose vaccination is indicated only during emergency response to YF outbreaks, when the shortage of YF-vaccine production or the need for the full-dose exceeds the capacity of the global stockpile. The WHO has developed an agenda to stimulate research to address policy-relevant issues. Critical questions still remain to be answered on the immunogenicity of fractional dose in young children (<2years), pregnant women, HIV-infected subjects as well as in immunocompromised patients. Moreover, a relevant issue is whether the immune responses to fractional dose are similar in populations with environmental exposures to other flaviviruses or flavivirus vaccination. Another gap refers to the lack of information about the long-term duration of immunogenicity and effectiveness of YF fractional vaccination as compared to the full dose (13).

Currently, there are a few world producers of YF vaccine prequalified by the World Health Organization (WHO) and the increasing demand for YF vaccine has become a challenge and impacted the international YF vaccine stockpile. Approximately 450 million doses are estimated to achieve high vaccination coverage (above $80 \%$ ) in areas of YF viral circulation but the annual production of YF vaccine accomplishes only 80 million doses, rendering at risk populations susceptible to the infection $(14,15)$. Considering the current YF epidemiological scenario worldwide, a set of measures has been proposed by the WHO to improve YF vaccine supply, including the recommendation of a dose sparing strategy, as a short-term measure (16). Roukens et al. (17) have proposed that intradermal administration of one fifth of the amount of YF vaccine results in seropositivity in all volunteers (17). In 2016, the Strategic Advisory Group of Experts (SAGE) from the WHO considered that the available evidences were sufficient to determine the use of fractional dose, as a safe and effective option for mass vaccination campaigns to control urban outbreaks in situations of YF vaccine shortage $(11,12,17)$.

Previous studies carried out by the Collaborative Group for Studies of Yellow Fever Vaccine (FIOCRUZ-Brazil) in 2009, as clinical dose-response cohort investigations with the 17DDYF vaccine $(18,19)$ have further contributed to support the use of lower dose of YF vaccine. Together, Martins et al. (18) and Campi-Azevedo et al. (19) demonstrated that doses above 3,013IU elicit similar levels of neutralizing antibodies, equivalent peak viremia and strong pro-inflammatory response in a similar timeline as compared to the reference full dose $(27,476 \mathrm{IU})$. Moreover, it was shown that the YF-specific immunity lasted up to 1 -year after primary vaccination with satisfactory levels of neutralizing antibodies $(18,19)$.

The knowledge about the long-term duration of YFspecific immunity after primary vaccination with lower doses is crucial to support and strengthen the use of fractional dose strategy. Recently, Martins et al. (18) have evaluated the status of YF-specific immunity in those participants on the dose-response study in 2009 and demonstrated that seropositivity was maintained in $85 \%$ of vaccinees across groups that received subdoses of $17 \mathrm{DD}-\mathrm{YF}$ vaccine (20). Moreover, Roukens et al. (21) have reported that intradermal administration of a one-fifth dose of 17D-YF vaccine induced a protective immune response that lasted for 10 years after vaccination (21).

The current study is a complementary investigation based on a parallel analysis of humoral and cellular immunity in a subset of volunteers originally enrolled in the doseresponse study in 2009. There is no precedent follow-up study that simultaneously evaluate the long-term duration of humoral and cellular immunity upon 17DD-YF vaccination with lower doses. These findings will add new evidences to support the regular use of dose sparing strategy for YF vaccine in adults.

\section{MATERIALS AND METHODS Study Population}

The present study was designed by the Collaborative Group for Studies of Yellow Fever Vaccine as an extension of the doseresponse study with the 17DD YF-vaccine conducted by BioManguinhos in 2009, to evaluate the YF-specific humoral and cellular immunity duration and to provide supportive evidences for the use of fractional doses. The original dose-response study carried out in 2009, when the volunteers were selected was a non-inferiority, double blind, randomized clinical trial of immunogenicity and safety. The current investigation is a 8 -years follow-up investigation that enrolled adult male army conscripts from Rio de Janeiro (a non-endemic area for YF in 2009), average age of 19.4 years old, who had received the reference full dose and subdoses of 17DD-YF vaccine during the dose-response study in 2009 (18). The target subjects were those with negative PRNT levels before vaccination in 2009 and who were not revaccinated. A total of 319 volunteers adhered to the current protocol and were eligible for the study. Exclusion criteria followed those described by Martins et al. (18), de Menezes Martins et al. (20) and briefly included: PRNT seropositivity at baseline in 2009, PRNT seronegativity at 30-45 days or 1-year upon primary vaccination and YF re-vaccination. Participants that had participated on military 
missions or traveled to sylvatic areas of YF viral circulation after 2009 were also excluded from the current investigation. Blood sampling was performed by qualified nursing team at FIOCRUZ, following informed consent and no access to additional clinical records was planned in the original study protocol. Before blood collection, participants were asked at least twice if they had been revaccinated. They were also asked to confirm that they had participated in the dose-response study in 2009. From the eligible population $(n=319), 98$ volunteers agreed to participate in this study and were categorized into six groups, according to the dose of 17DD-YF vaccine administered in 2009: $27,476 \mathrm{IU}$, considered as the reference full dose $(n=16)$; $10,447 \mathrm{IU}(n=17) ; 3,013 \mathrm{IU}(n=19) ; 587 \mathrm{IU}(n=17)$; $158 \mathrm{IU}$ $(n=18)$ and $31 \mathrm{IU}(n=11)$. In the original study, a single dose of $0.5 \mathrm{~mL}$ of 17DD-YF vaccine formulations with decreasing amounts of viral particles was given to each participant. An additional group of 46 adult male army conscripts from a database of another study carried out by our own group (22) was included as non-vaccinated controls and referred as NV (day0). Detailed compendium of the study population and methods are provided in the study design flowchart showed in the Figure 1.
Whole blood samples were collected from each volunteer, including $5 \mathrm{~mL}$ in tubes without anticoagulant for YF-plaque reduction neutralization test (PRNT) and $20 \mathrm{~mL}$ in heparin sodium for 17DD-YF phenotypic and functional analyses of cellular immunity profile.

This is an 8-years follow-up study included in a clinical trial registry (NCT 03338231). The study protocol was approved by the Ethics Committee at Instituto Nacional de Infectologia Evandro Chagas, FIOCRUZ (Plataforma Brasil, CAAE\#65823617.6.3001.5091). All procedures followed the Helsinki Declaration, the Brazilian ethical standards of scientific research involving human subjects and the good clinical practices.

\section{Serology for YF-Plaque Reduction Neutralizing Test (PRNT)}

The PRNT levels to the 17DD-YF virus were quantified using the same method employed in the dose-response study of 2009 (18), using the same cut-off for seropositivity: $>2.7 \log _{10} \mathrm{mIU} / \mathrm{mL}$ (501.2 $\mathrm{mIU} / \mathrm{mL}$ ), or about $1 / 20$ in dilution. The PRNT analysis was performed at Laboratório de Tecnologia Virológica, BioManguinhos (LATEV, FIOCRUZ-RJ, Brazil).

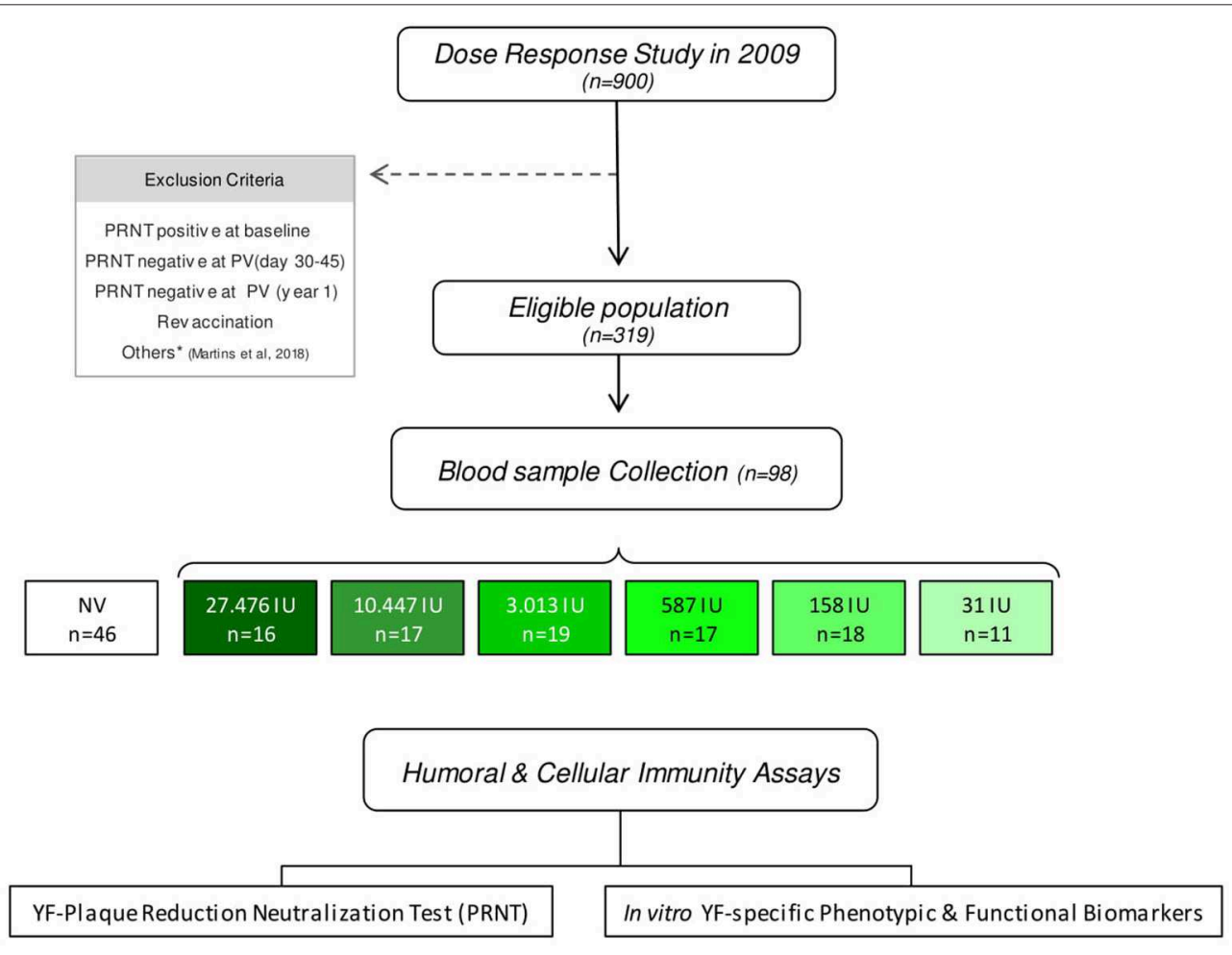

FIGURE 1 | Study design flowchart. The consort diagram summarizes the study steps. This is a 8-years follow-up study, of adult male army conscripts from Rio de Janeiro, average age of 19.4 years old, who had received the reference full dose and subdoses of 17DD-YF vaccine during the dose-response study in 2009 (12). From the eligible population $(n=319)$, only 98 volunteers agreed to provide two blood samples, one without anticoagulant for humoral analysis and an additional heparinized sample for cellular immunity assays. These volunteers were categorized into six groups, according to the dose of 17DD-YF vaccine administered in 2009: $27,476 \mathrm{IU}$, considered the reference dose $(\square, n=16) ; 10,447 \mathrm{IU}(\square, n=17) ; 3,013 \mathrm{IU}(\square, n=19) ; 587 \mathrm{IU}(\square, n=17) ; 158 \mathrm{IU}(\square, n=18)$, and $31 \mathrm{IU}(\square, n=11)$. An additional group of non-vaccinated adult male army conscripts, referred as NV(day0), was included as a control ( $\square, n=46$ ). Humoral and cellular immunity profile was determined for each volunteer using the YF-plaque reduction neutralization test (PRNT) and in vitro YF-specific phenotypic \& functional biomarkers. 


\section{In vitro Assays for YF-Specific Phenotypic and Functional Memory Biomarkers}

The peripheral blood lymphoproliferation assay for measuring YF-specific cellular immunity memory was performed as previously described by Costa-Pereira et al. (22). Briefly, aliquots $\left(1.0 \times 10^{6} /\right.$ well $)$ of peripheral blood mononuclear cells (PBMC) were incubated in the absence (Control) or presence of 17DD$\mathrm{YF}$ vaccine stimuli (17DD-YF Ag), at $37^{\circ} \mathrm{C}$ in a $5 \% \mathrm{CO}_{2}$ for 6 days. After incubation, PBMC were harvested and stained with live/dead dye (Life Technologies, Carlsbad, CA, USA) and a mix of monoclonal antibodies (mAbs) [anti-CD4/(RPA-T4)/FITC; anti-CD8/(SK1)/PerCP-Cy5.5; anti-CD27/(M-T271)/PE, anti$\mathrm{CD} 45 \mathrm{RO} /(\mathrm{UCHL} 1) / \mathrm{PE}-\mathrm{Cy} 7$ and anti-CD3/(SK7)/APC-Cy7] to quantify memory T-cell subsets and [anti-CD19/(HIB19)/PerCP, anti-CD27/(M-T271)/PE and anti-IgD/(IA6-2)/FITC] for B-cell analysis. All mAbs were purchased from BD Pharmingen (BD Bioscience, San Diego, CA, USA).

Additional aliquots of cultured PBMC were labeled with live/dead dye and a cocktail of mAbs [antiCD3/(UCHT1)/Qdot605 (Invitrogen, Carlsbad, CA, USA); anti-CD4/(GK1.5)/APCe-Fluor780 (eBioscience, San Diego, CA, USA); anti-CD8/(SK1)/PerCP (BD Biosciences, San Diego, CA, USA) and anti-CD19/(HIB19)/Alexa-Fluor700 (eBioscience, San Diego, CA, USA)] to identify T-cell subsets and B-cells. Following the Fix/Perm step, cells were reincubated with a mix of mAbs [anti-TNF- $\alpha /($ clone MAb11)/PE-Cy7; anti-IFN- $\gamma /($ clone
B27)/Alexa-Fluor488); anti-IL-5/(JES1-39D10)/PE and antiIL-10/(JES3-19F1)/APC, all purchased from BD Bioscience] to enumerate functional status of $\mathrm{T}$ and $\mathrm{B}$-cells.

Stained PBMC were fixed and stored at $4^{\circ} \mathrm{C}$ up to $24 \mathrm{~h}$ prior to flow cytometric acquisition on a BD LSR Fortessa (BD Bioscience, San Diego, CA, USA).

A total of 100,000 events were acquired per each sample and data stored for offline analysis. The FlowJo software (version 9.3.2, TreeStar, San Diego, CA, USA) was employed for data analysis. The expression of CD45RO and CD27 was used to define memory $\mathrm{CD} 4^{+}$and $\mathrm{CD} 8^{+}$T-cell subsets: Naïve/NCD4;NCD8 - $\left(\mathrm{CD} 27^{+} \mathrm{CD} 45 \mathrm{RO}^{-}\right)$; early Effector/eEfCD4;eEfCD8 - $\left(\mathrm{CD} 27^{-} \mathrm{CD}^{2} 5 \mathrm{RO}^{-}\right)$; Central Memory/CMCD4;CMCD8 - $\left(\mathrm{CD} 27^{+} \mathrm{CD}^{2} 5 \mathrm{RO}^{+}\right)$and Effector Memory/EMCD4;EMCD8 - $\left(\mathrm{CD} 27^{-} \mathrm{CD} 45 \mathrm{RO}^{+}\right)$. The expression of $\operatorname{IgD}$ and $\mathrm{CD} 27$ was employed to define memory B-cell subsets: Naïve/NCD19 - $\left(\mathrm{CD} 27^{-} \mathrm{IgD}^{+}\right)$; Nonclassical Memory/nCMCD19 - $\left(\mathrm{CD}_{27}{ }^{+} \mathrm{IgD}^{+}\right)$and Classical Memory/CMCD19 - $\left(\mathrm{CD} 27^{+} \mathrm{IgD}^{-}\right)$. Functional $\mathrm{CD}^{+}$and $\mathrm{CD}^{+}$T-cell subsets (TNF- $\alpha$, IFN- $\gamma$, IL-10, and IL-5) as well as B-cells (TNF- $\alpha$, IL-10, and IL-5) were also quantified. The results were first generated as percentage of memory $\mathrm{T}$ and B-cell subsets in parallel with cytokine ${ }^{+}$-cells. The final results of YF-specific phenotypic and functional memory biomarkers were presented as 17DD-YF Ag/Control culture Index, calculated as the ratio between the percentages of cells observed in the

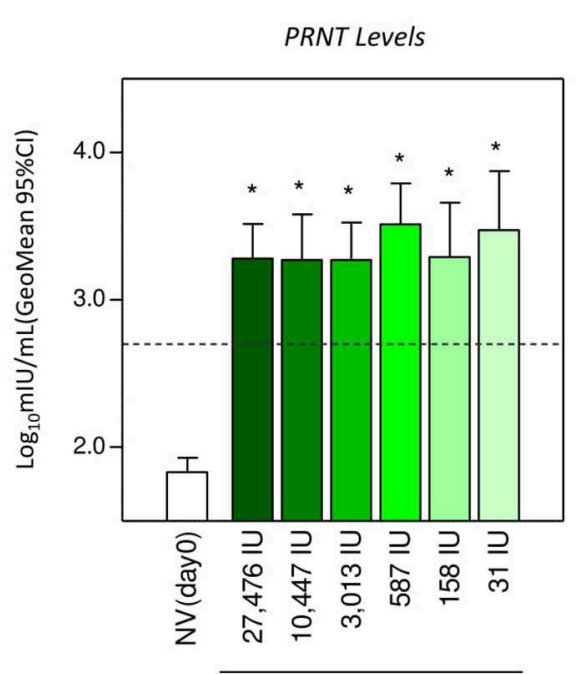

PV(year8)

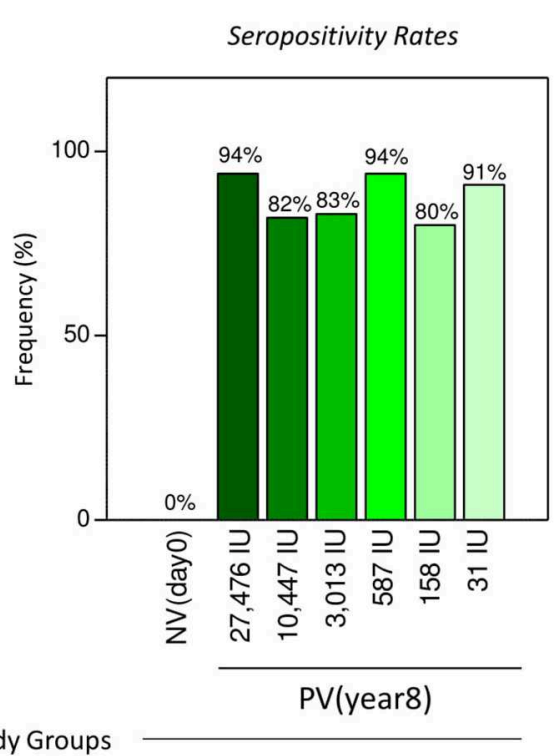

FIGURE 2 | Neutralizing antibodies levels and PRNT seropositivity rates 8-years after 17DD-YF primary vaccination with different doses. The YF-specific plaque reduction neutralizing antibodies were measured using the same method employed in the dose-response study of 2009 (12). The cut-off for seropositivity was set at $>2.7 \log _{10} \mathrm{mlU} / \mathrm{mL}(501.2 \mathrm{mlU} / \mathrm{mL})$ or about $1 / 20$ in dilution. The eligible vaccinees $(n=98)$ were categorized into six subgroups, according to the dose of $17 \mathrm{DD}-\mathrm{YF}$ vaccine administered in 2009: 27,476IU, considered the reference dose $(\square, n=16) ; 10,447 \mathrm{IU}(\square, n=17) ; 3,013 \mathrm{IU}(\square, n=18) ; 587 \mathrm{IU}(\square, n=17) ; 158 \mathrm{IU}(\square, n=15)$, and $31 \mathrm{IU}(\square, n=11)$. A group of non-vaccinated adult male army conscripts, referred as NV(day0), was included as a control ( $\square, n=46)$. The PRNT levels were expressed as geometric mean titer and $95 \% \mathrm{Cl}$ of $\log _{10} \mathrm{mlU} / \mathrm{mL}$ and the seropositivity rates shown as frequency (\%) of subjects above the cut-off edge $\left(2.7 \log _{10}\right.$ $\mathrm{mlU} / \mathrm{mL}$ - dashed line). Comparative analysis of PRNT levels and seropositivity rates were assessed by ANOVA adjusted to multiple comparisons and Fisher exact test, respectively. In all cases, significant differences were considered at $p<0.05$ and highlighted by asterisk (*) as compared to NV(day0). 
17DD-YF Ag cultures by percentage observed in the respective control culture.

\section{Statistical Analysis}

Statistical analysis was performed, first blindly, by using ANOVA adjusted to multiple comparisons and Fisher's exact test, as indicated. After code unblinding, each 17DD-YF vaccine group was compared to the control group of non-vaccinated subjects $-\mathrm{NV}($ day0). The iterative proportion fitting of PRNT seropositivity rates were also calculated for comparative analysis amongst groups. In all cases, significant differences were considered at $p<0.05$ and highlighted by asterisk $\left(^{*}\right)$ as compared to $\mathrm{NV}($ day0).

\section{RESULTS}

\section{Similar Immunogenicity Is Maintained 8-Years After 17DD-YF Primary Vaccination With Lower Doses}

The geometric mean titers of YF-specific plaque reduction neutralizing antibodies and the frequency of seropositive vaccinees $\left(>2.7 \quad \log _{10} \mathrm{mIU} / \mathrm{mL}\right)$ at 8 years upon 17DD-YF primary vaccination with different doses are presented in the Figure 2. The 8-years followup study demonstrated that volunteers, who had seroconverted upon 17DD-YF primary vaccination with lower doses and had not been revaccinated, still presented

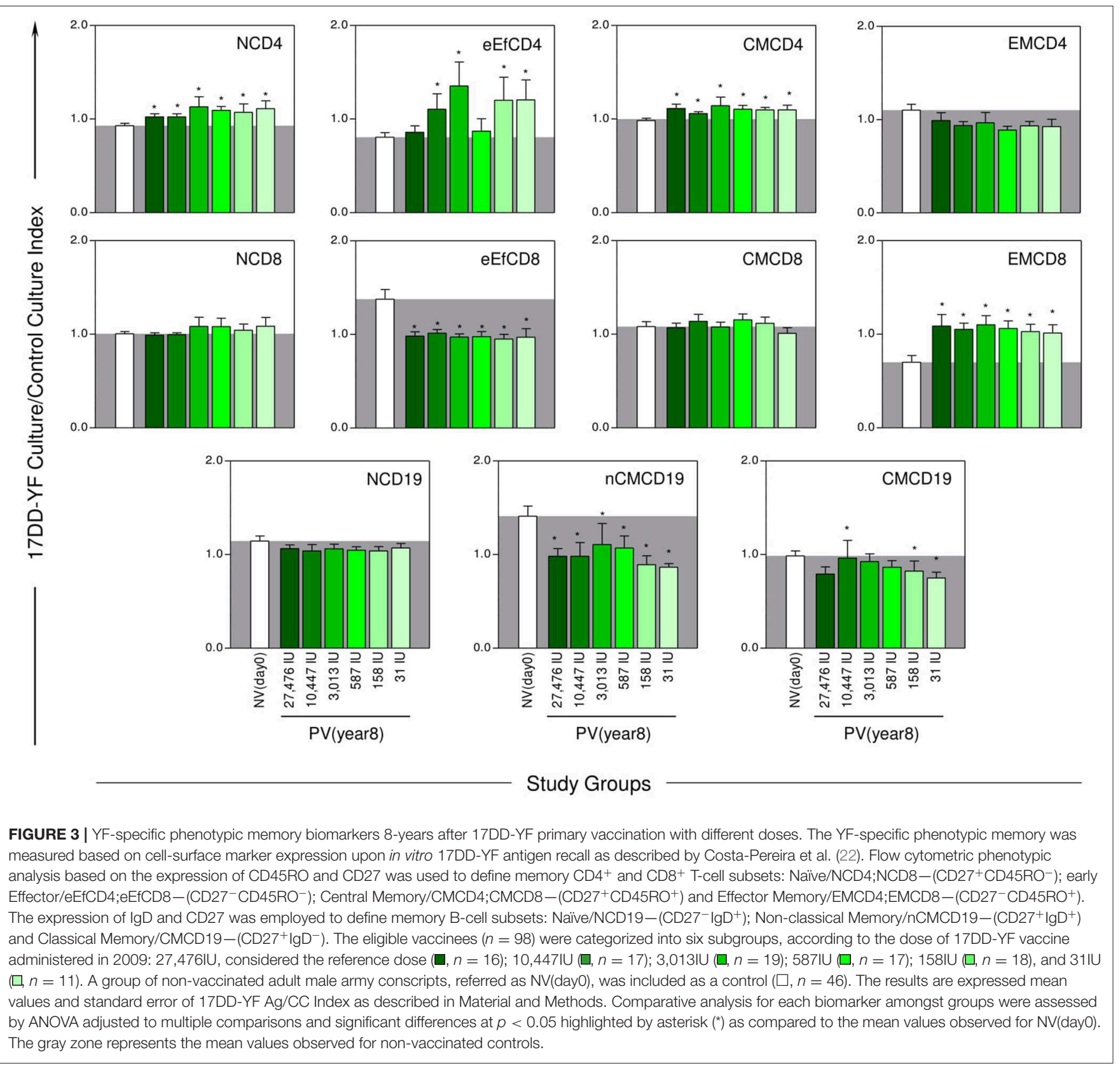




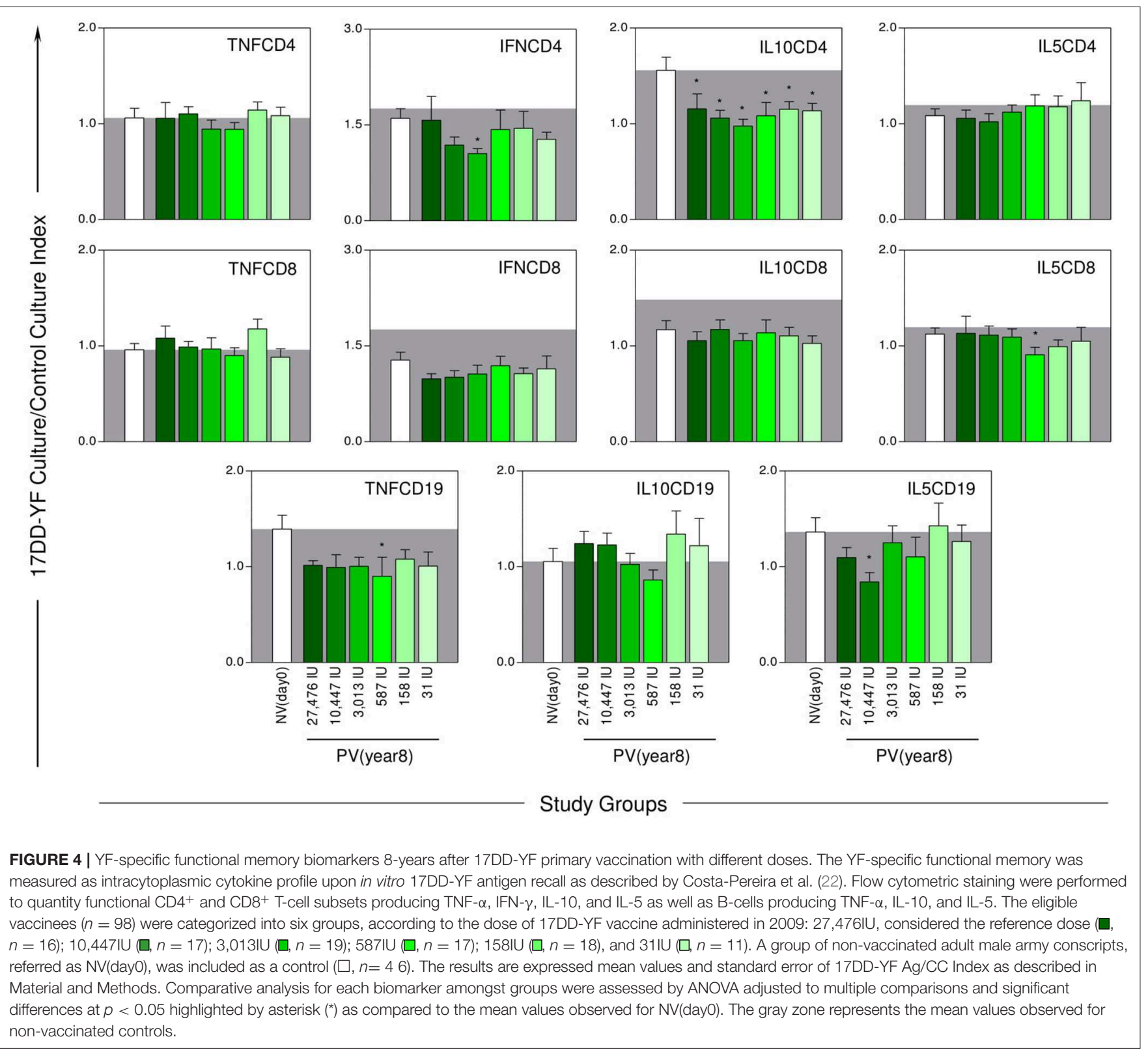

similar antibody levels and seropositivity rates as in all subdoses administrated as compared to the reference full dose (Figure 2).

Aiming to determine the overall seropositivity rates achieved by the primary vaccination with lower doses of 17DD-YF vaccine, since the seroconversion at 30-45 days throughout the 8 -years time span follow-up, an iterative proportion fitting of PRNT seropositivity rates was calculated as a progressive decrease on seropositivity rates along time and data presented in the Supplementary Figure 1. The results demonstrated that at 30-45 days, doses from 27,476IU (reference full dose) above $587 \mathrm{IU}$ displayed similar immunogenicity $(96,100,97$, and $97 \%$, in that order), while doses of $158 \mathrm{IU}$ and $31 \mathrm{IU}$ induced lower PRNT levels (89 and 56\%, respectively). The 1-year follow-up included only volunteers who had seroconverted at day 30-45 after vaccination and had not been re-vaccinated. Seropositivity was maintained up to 1-year across doses (99, 97, 99, 100, and 97\%, correspondingly), except for the $31 \mathrm{IU}$ group that lead to $89 \%$ of seropositivity. The present study, carried out 8 years after primary vaccination, included only volunteers who had seroconverted at day 30-45 after vaccination, maintained the seropositivity at 1-year and had not been revaccinated. Subjects with negative PRNT at 3045 days or 1-year after primary vaccination were not included in the present investigation since they were re-vaccinated as part of the study protocol approved in 2009. The results 
demonstrated an overall PRNT seropositivity rate of $87 \%$, with similar rates across groups $(94,82,83,94,80$, and $91 \%$, correspondingly). However, the iterative proportion fitting of seropositivity rates demonstrated that only doses above 587IU elicited similar immunogenicity $(89,80,80$, and $91 \%$, respectively), whereas doses of $158 \mathrm{IU}$ and $31 \mathrm{IU}$ reached lower proportion of seropositivity rates (68 and $46 \%$, in that order) (Supplementary Figure 1).

\section{Equivalent YF-Specific Cellular Memory Is Observed 8-Years After 17DD-YF Primary Vaccination With Lower Doses}

The YF-specific phenotypic and functional memory profiles at 8 -years after 17DD-YF primary vaccination with different doses are shown in the Figures 3, 4. The YF-specific memory biomarkers were assessed upon in vitro $17 \mathrm{DD}-\mathrm{YF}$ antigen recall and the results presented as 17DD-YF Ag/Control culture Index.

Analysis of phenotypic memory biomarkers revealed, regardless of the $17 \mathrm{DD}-\mathrm{YF}$ dose used at primary vaccination, higher levels of NCD4 in all vaccinees as compared to the $\mathrm{NV}$ (day0) control group. Conversely, lower levels of eEfCD8 and nCMCD19 were similarly observed across groups as compared to the $\mathrm{NV}$ (day0) control group. Waves of eEfCD 4 were observed in 4 out of 5 lower doses tested (10,447IU, 3,013IU, 158IU, and 31IU). Three particular profiles were observed for CMCD19, including higher levels in 10,447IU and lower levels in 158IU and 31IU groups. Of note were the increased levels of CMCD4 and the remarkable levels of EMCD8 observed in all vaccinees even 8-years upon 17DD-YF primary vaccination with lower doses (Figure 3).

The data from the current investigation was compared with those from another study carried out by our own group, in which the participants received the "routine" full dose of 17DD-YF vaccine (13). In both studies the results were obtained using the same methods to quantify the levels of neutralizing antibodies and EMCD8. Using the combined database from both studies, the YF-specific PRNT and EMCD8 profiles were compared and the results presented in the Supplementary Figure 2. Data analysis demonstrated that 8-years after primary vaccination with17DDYF subdoses, all selected vaccinees still presented preserved levels of PRNT and EMCD8, similar to those observed in PV(day3045) and PV(year7-9) but higher than that observed in NV(day0) and $\mathrm{PV}($ year $>10)$.

The analysis of functional biomarkers demonstrated equivalent lower levels of IL10CD4 across groups. Several random point profiles, such as lower levels of IL5CD19, IFNCD4 along with TNFCD19 and IL5CD8, were observed in 10,447IU, 3,013IU, and 587IU groups, respectively (Figure 4 ).

Together, these findings demonstrated that volunteers, who had seroconverted upon $17 \mathrm{DD}-\mathrm{YF}$ primary vaccination with lower doses and had not been revaccinated, still presented in an 8-years follow-up study comparable YF-specific cellular memory, particularly CMCD4 and EMCD8 as compared to the reference full dose (Figure 3).

\section{Comparable Biomarker Network Portrait Is Perceived 8-Years After 17DD-YF Primary Vaccination With Different Doses}

Biomarker networks were built to define connections between YF-specific humoral and cellular memory at 8-years follow 17DD-YF primary vaccination with different doses (Figure 5). In general, there was a predominance of positive correlations " $r(+) / r(-)$ " between YF-specific humoral and cellular memory at 8 -years upon primary vaccination in most subgroups, with higher ratios for doses of 27,476IU, 10,447IU, and 3,013IU (31/18, $15 / 11$, and 21/11, respectively). Strong negative correlations were observed for "EMCD4,1/NCD4" and "EMCD8,1/NCD8" in all groups, regardless of the dose administrated. Noteworthy was the strong positive correlation observed for "EMCD4,EMCD8" and "TNFCD8,IFNCD8" in most groups, except for volunteers receiving the $31 \mathrm{IU}$ dose (Figure 5).

\section{Analogous Snapshot Highlights the Major Similarities of YF-Specific Humoral and Cellular Memory Profile 8-Years After 17DD-YF Primary Vaccination With Different Doses}

The selection of biomarkers presenting common profiles throughout distinct doses of 17DD-YF vaccine is compiled in the Figure 6. Humoral immunogenicity, phenotypic and functional cellular memory and correlation links between attributes were assembled to identify universal biomarkers across the groups. Arrows were employed to highlight decreased $(\downarrow)$ or increased levels $(\uparrow)$ as compared to NV(day0). Data analysis revealed that a range of attributes ( $\uparrow$ PRNT, $\uparrow N C D 4, \uparrow C M C D 4$, $\downarrow$ IL10CD4, $\downarrow$ eEfCD8, $\uparrow$ EMCD8, $\downarrow$ nCMCD19) presented similar profiles throughout the distinct 17DD-YF vaccine doses even 8years after primary vaccination (Figure 6). Moreover, negative correlations between "EMCD4,1/NCD4" and "EMCD8,1/NCD8" along with positive correlation for "EMCD4,EMCD8" and “TNFCD8,IFNCD8" were also observed. Worth mentioning was the preserved PRNT titers, the gold standard to measure postvaccination immunity to $\mathrm{YF}$, and the outstanding levels of EMCD8, a relevant correlate of protection for YF-specific cellular immunity (Figure 6).

\section{DISCUSSION}

The current study is a complementary investigation that simultaneously evaluate the long-term duration of humoral and cellular immunity in a subset of volunteers originally receiving subdoses of $17 \mathrm{DD}-\mathrm{YF}$ vaccine, enrolled in the dose-response study in 2009.

Few studies have addressed the duration of immunity after immunization with reduced doses of YF vaccine. The fractional dose of 17DD-YF vaccine has been shown to be safe and noninferior to the standard dose in inducing seroprotection (17-19). The seropositivity rates reported by Ahuka-Mundeke et al. (23) in a recent vaccination campaign using one-fifth fractional-dose in Africa are similar to those obtained by Martins et al. (18) and 


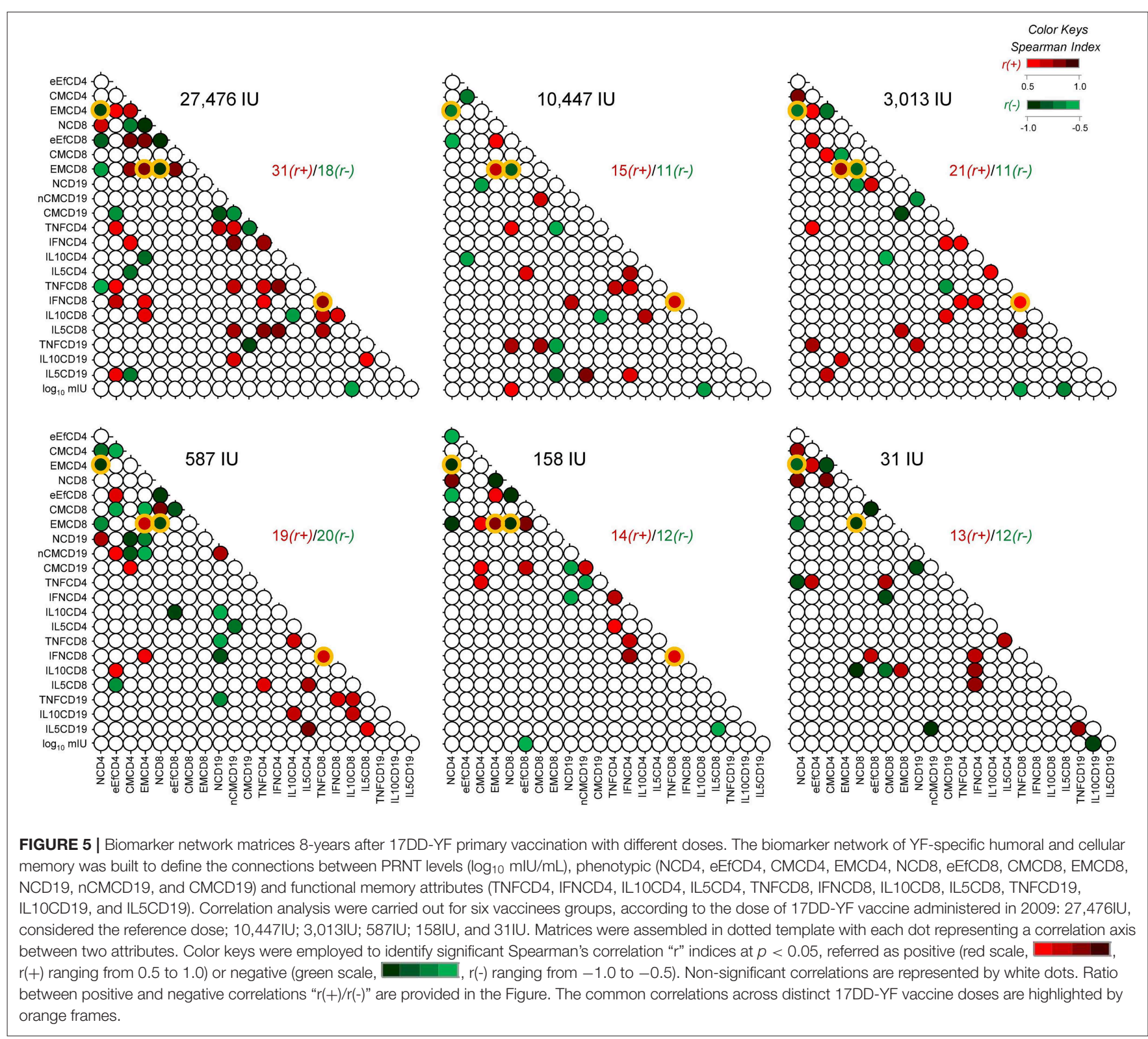

Campi-Azevedo et al. (19), showing 98\% seroconversion at 3045 days after primary vaccination in seronegative subjects (23). A long-term follow-up randomized, controlled, non-inferiority trial study conducted with the 17D-YF vaccine has shown that a one-fifth dose YF vaccine induced a protective immunity that lasted for 10 years after vaccination (21).

Recently, Martins et al. (18) have evaluated the duration of immunity by measuring neutralizing antibody levels 8 -years after the dose-response study conducted in 2009 using subdoses of 17DD-YF vaccine. Seropositivity was maintained in $85 \%$ of 318 participants and was similar across groups as compared to the reference full dose. The present results demonstrated that, after 8-years, subjects who had seroconverted after 17DDYF primary vaccination still presented an overall seropositivty rate of $87 \%$, similar to that observed by Martins et al. (18).
Vaccinees who received doses from 10.447IU down to 31 IU still presented high seropositivity rates $(82,83,94,80$, and $91 \%$, in that order) comparable to that reported for the reference full dose (94\%). It is important to mention that only volunteers who had seroconverted at 30-45 days after primary vaccination in 2009 and maintained the seropositivity at 1-year with no records of revaccination have been included in the present investigation. However, in order to overcome this putative drawback, the overall seropositivity has been calculated as iterative proportion fitting of PRNT seropositivity rates since the seroconversion at 30-45 days throughout the 8-years time span follow-up. The data demonstrated that doses higher then 587IU displayed similarly high immunogenicity while doses of 158IU and 31IU induced lower PRNT levels. Which was a direct reflection of the higher failure of primary seroconversion at 30-45 days observed for the 


\begin{tabular}{|c|c|c|c|c|c|c|c|}
\hline & & 27,476 IU & $10,447 \mathrm{IU}$ & 3,013 IU & $587 \mathrm{IU}$ & $158 \mathrm{IU}$ & $31 \mathrm{IU}$ \\
\hline & 을 & ^PRNT & ^PRNT & ^PRNT & 个PRNT & 个PRNT & ^PRNT \\
\hline & $\overline{\widetilde{\sigma}}$ & $\uparrow N C D 4$ & $\uparrow N C D 4$ & $\uparrow N C D 4$ & $\uparrow N C D 4$ & $\uparrow N C D 4$ & $\uparrow N C D 4$ \\
\hline & 으 & $\uparrow$ CMCD4 & $\underline{\uparrow C M C D 4}$ & 1 CMCD4 & ^CMCD4 & $\underline{\uparrow C M C D 4}$ & ^CMCD4 \\
\hline & & $\underline{\downarrow} \underline{\mathrm{IL} 10 \mathrm{CD} 4}$ & $\downarrow$ IL10CD4 & $\downarrow$ IL10CD4 & $\downarrow$ IL10CD4 & $\downarrow \mathrm{IL} 10 \mathrm{CD} 4$ & $\downarrow$ IL10CD4 \\
\hline 는 & Ш ర & & & & & & \\
\hline$\stackrel{2}{2}$ & $0 \frac{5}{0}$ & $\frac{\text { DEETCDS }}{\text { TFMCDR }}$ & $\checkmark$ eEfCD 8 & $\downarrow$ eEfCD 8 & $\checkmark$ eEfCD8 & עeEfCD 8 & $\checkmark$ eEfCD 8 \\
\hline$\frac{y}{2}$ & $\sum^{2}$ & & & & - & & \\
\hline 듬 & & $\downarrow$ nCMCD 19 & $\downarrow$ nCMCD19 & $\downarrow \mathrm{nCMCD19}$ & $\downarrow$ nCMCD 19 & $\downarrow$ nCMCD 19 & $\downarrow$ nCMCD 19 \\
\hline & $\stackrel{0}{\complement}$ & EMCD4,1/NCD4 & EMCD4,1/NCD4 & EMCD4, 1/NCD4 & EMCD4,1/NCD4 & EMCD4V1/NCD4 & EMCD4, 1/NCD4 \\
\hline & స్తు & EMCD8, $1 /$ NCD8 & EMCD8, $1 /$ NCD8 & EMCD8, 1 /NCD8 & EMCD $8,1 / \mathrm{NCD} 8$ & EMCD8, 1/NCD8 & EMCD8, $1 /$ NCD8 \\
\hline & Ф) & TNFCD8, IFNCD8 & $\underline{\text { TNFCD } 8, \text { IFNCD } 8}$ & $\underline{\text { TNFCD } 8, \text { IFNCD } 8}$ & $\underline{\text { TNFCD8, IFNCD8 }}$ & $\underline{\mathrm{TNFCD} 8}, \underline{\mathrm{IFNCD} 8}$ & \\
\hline & (7) & EMCD4,EMCD8 & EMCD4,EMCD8 & EMCD4, EMCD8 & EMCD4,EMCD8 & EMCD4,EMCD8 & \\
\hline
\end{tabular}

\section{DD-YF Vaccine Doses}

FIGURE 6 | Snapshot overview of major similarities of YF-specific humoral and cellular memory profile 8-years after 17DD-YF primary vaccination with different doses. Selection of biomarkers with similar profile throughout distinct subdoses of 17DD-YF vaccine (27,476IU - reference dose; 10,447IU; 3,013IU; 587IU; 158IU and 31IU). Humoral memory immunity $(\dot{\psi}=$ PRNT) was measured by YF-specific plaque reduction neutralizing antibodies using the same method employed in the dose-response study of 2009 (9). The YF-specific phenotypic and functional memory biomarkers $(\Theta=$ NCD4, CMCD4, and IL10CD4; $\bigcirc=$ eEfCD8 and EMCD8 along with $0=$ nCMCD19) were measured upon in vitro 17DD-YF antigen recall as described by Costa-Pereira et al. (22). Arrows highlighted decreased ( $\downarrow$ ) or increased levels ( $\uparrow$ ) as compared to NV(day0). Correlation analysis were carried out to identify significant negative ( 0 = "EMCD4,1/NCD4" and $\boldsymbol{Q}=$ = "EMCD8,1/NCD8") or positive $(\mathcal{C O}=$ "TNFCD8,IFNCD8" and $\mathbb{C}$ = "EMCD4,EMCD8") common correlations throughout distinct subdoses of 17DD-YF vaccine (27,476IU - reference dose; 10,447IU; 3,013IU; 587IU; 158IU and 31IU), except the correlations "TNFCD8,IFNCD8" and "EMCD4,EMCD8" not observed for the lower dose (31IU).

two lowest doses (158IU and 31IU) $(18,19)$. Therefore, these doses should be considered inferior to the other doses tested.

The results also pointed out that biological markers of memory for cellular immunity, particularly EMCD8, were found in comparable levels across groups. Noteworthy was the strong positive correlations ("EMCD4,EMCD8" and "TNFCD8,IFNCD8") observed in most subdoses, except for 31IU. A predominance of positive correlations between YFspecific humoral and cellular memory was observed in most groups at 8-years upon primary vaccination, with higher ratios found for doses 27,476IU, 10,447IU, and 3,013IU. Moreover, the high number of connectivity triggered by the reference full vaccine dose $(27,476 \mathrm{IU})$ may represent the massive activation of a wide range of cell clones elicited by the high antigenic concentration. Although the number of connectivity drops significantly in lower YF vaccine doses, the correlates of protection (PRNT and EMCD8) still remains detectable in similar levels across all doses. These findings allude to those previously reported by Campi-Azevedo et al. (19) that higher similarities in immunological and virological parameters were found for subdoses down to $3,013 \mathrm{IU}$ as compared to the reference full dose $(27,476 \mathrm{IU})$, while lower subdoses elicited an impaired magnitude of equivalence. Therefore, the biomarkers associated the preserved connections such as "EMCD4,EMCD8", "TNFCD8,IFNCD8" besides "EMCD4,1/NCD4" and "EMCD8,1/NCD8" might represent relevant axis to guarantee the protective immunity upon 17DD-YF vaccination.

Major similarities underscored the preserved PRNT titers and the outstanding levels of EMCD8, relevant correlates of protection for YF-specific immunity. The neutralizing antibodies levels have been considered the convenient proxy correlate of protection to monitor the immunogenicity of YF vaccination (3). Several studies have demonstrated that neutralizing antibodies and YF-specific $\mathrm{CD} 8^{+} \mathrm{T}$-cell are relevant imprints of immunological memory induced by YF vaccination 
(22, 24-28). The protective immune memory developed after YF vaccination comprises the ability to produce YFneutralizing antibodies together with the generation of effector memory $\mathrm{CD}^{+}$T-cell. The high magnitude, broad specificity, robust proliferative profile, multiple functions and long-term persistence of immune memory mediated by $\mathrm{CD} 8^{+}$T-cells is an attribute that defines efficacious immune response after vaccination (29). Focusing on these two premium biomarkers (PRNT and EMCD8), an additional analysis was performed using a combined database from the current investigation and from another study of our own group using the "routine" full dose of $17 \mathrm{DD}-\mathrm{YF}$ vaccine (22). The results further supports that 8 -years after primary vaccination with17DD-YF subdoses all selected vaccinees still presented preserved levels of correlates of protection.

Together, the current findings demonstrated that the duration of immunity upon vaccination of adults with subdoses of 17DDYF vaccine is acceptable and comparable with that observed for the reference full dose, except for 158IU and 31IU groups that elicited lower iterative proportion of PRNT seropositivity rates along time. The PRNT levels and seropositivity rates along with the profile of EMCD8 provide relevant evidences to support the use of dose sparing strategy for YF vaccine in adults.

Dose-response studies are still required for its universal use in children $<2$-years of age, elderly, pregnant women as well as immunocompromised patients considering the particularities of their immune response. Of our knowledge, recent studies are currently under investigation in Africa to evaluate fractional dose YF vaccination in children, adults and HIV-infected subjects. A dose-response study in children has been considered by our group and has being submitted for evaluation. The limited data on duration of protection also does not qualify people for international travel under the International Health to receive fractional dose vaccination.

\section{DATA AVAILABILITY}

All datasets generated for this study are included in the manuscript and/or the Supplementary Files.

\section{ETHICS STATEMENT}

This study was carried out in accordance with the recommendations of Brazilian ethical standards of scientific

\section{REFERENCES}

1. Douam F, Ploss A. Yellow fever virus: knowledge gaps impeding the fight against an old foe. Trends Microbiol. (2018) 26:913-28. doi: 10.1016/j.tim.2018.05.012

2. Monath TP, Vasconcelos PFC. Yellow fever. J Clin Virol. (2015) 64:160-73. doi: 10.1016/j.jcv.2014.08.030

3. Collaborative Group for Studies on Yellow Fever Vaccines. Duration of post-vaccination immunity against yellow fever in adults. Vaccine. (2014) 32:4977-84. doi: 10.1016/j.vaccine.2014.07.021

4. Campi-Azevedo AC, Costa-Pereira C, Antonelli LR, Fonseca CT, Teixeira-Carvalho A, Villela-Rezende G, et al. Booster dose after research involving humans and the good clinical practices with written informed consent from all subjects. All subjects gave written informed consent in accordance with the Declaration of Helsinki. The protocol was approved by the Ethics Committee at Instituto Nacional de Infectologia Evandro Chagas, FIOCRUZ (Plataforma Brasil, CAAE\#65823617.6.3001.5091).

\section{AUTHOR CONTRIBUTIONS}

AC-A, MM, LC, RF, RM, AH, AR, CD, AT-C, and OM-F: designing research study. RM, OM-F, AR, and $\mathrm{CD}$ : funding acquisition. IC-R, AC-A, VP-M, JC-d-R, JF, TS-L, LR, LF, CC-P, SL, AT-C, and OM-F: conducting experiments. MM, TN, JX, EA, RF, and TC: field study. AC-A, VP-M, JC-d-R, JF, and SL: acquiring data. IC-R, AC-A, LF, JM, AT-C, and OM-F: analyzing data. TN, JX, EA and TC: validation. $\mathrm{RM}$ and $\mathrm{AH}$ : advisory committee. IC-R, AC-A, JC-d-R, CC-P, LR, JM, AT-C and OM-F: writing the manuscript.

\section{ACKNOWLEDGMENTS}

This study was funded by a grant from Wellcome Trust (grant\#206523/Z/17/Z) and Programa Nacional de Imunizações (PNI/SVS), Ministério da Saúde do Brasil (Fundo Nacional da Saúde, TED 205/2017, 29/12/2017). The research was also supported by Fundação de Amparo à Pesquisa do Estado de Minas Gerais (FAPEMIG), Biomanguinhos/FIOCRUZ, PROEP/CPqRR/FIOCRUZ, Conselho Nacional de Desenvolvimento Científico e Tecnológico - CNPq. The authors thank the Program for Technological Development in Tools for Health-RPT-FIOCRUZ for using the flow cytometry facilities. The authors acknowledge the Programa de Pós-Graduação em Ciências da Saúde do Instituto René Rachou - FIOCRUZMinas, supported by the Coordenação de Aperfeiçoamento de Pessoal de Nível Superior (CAPES) and the Programa PRINT-FIOCRUZ-CAPES. LC, OM-F, AT-C received PQ fellowships from CNPq.

\section{SUPPLEMENTARY MATERIAL}

The Supplementary Material for this article can be found online at: https://www.frontiersin.org/articles/10.3389/fimmu. 2019.01211/full\#supplementary-material

10 years is recommended following 17DD-YF primary vaccination. Hum Vaccines Immunother. (2016) 12:491-502. doi: 10.1080/ 21645515.2015

5. World Health Organization. Data From: In Disease Outbreak News - Yellow fever - Republic of the Congo. (2018). Available online at: https://www.who. int/csr/don/7-september-2018-yellow-fever-congo/en/ (accessed February 2019).

6. The Lancet. Yellow fever: a major threat to public health. Lancet. (2018) 391:402. doi: 10.1016/S0140-6736(18)30152-1

7. Almeida MA, Dos Santos E, da Cruz Cardoso J, da Fonseca DF, Noll CA, Silveira VR, et al. Yellow fever outbreak affecting Alouatta populations in southern Brazil (Rio Grande do Sul 
State), 2008-2009. Am J Primatol. (2012) 74:68-76. doi: 10.1002/ ajp. 21010

8. Romano APM, Costa ZGA, Ramos DG, Andrade MA, Jayme VdS, Almeida MA, et al. Yellow fever outbreaks in unvaccinated populations, Brazil, 2008- 2009. PLoS Neglect Trop Dis. (2014) 8:e2740. doi: 10.1371/ journal.pntd.0002740

9. Ministério da Saúde, Secretaria de Vigilância em Saúde. Data From: Reemergência da Febre Amarela Silvestre no Brasil, 2014/2015: Situação Epidemiológica e a Importância da Vacinação Preventiva e da Vigilância Intensificada no Período Sazonal. Boletim Epidemiológico Volume $46 \mathrm{~N}^{\circ}$ 29 (2015) Available online at: http://portalarquivos2.saude.gov.br/images/pdf/ 2015/outubro/19/2015-032---FA-ok.pdf.

10. Ministério da Saúde, Secretaria de Vigilância em Saúde. Data From: Emergência Epidemiológica de Febre Amarela no Brasil, no Período de Dezembro de 2016 a Julho de 2017. Boletim Epidemiológico Vol. 48 - $\mathrm{N}^{\circ}$ 28 (2017) Available online at: http://portalarquivos2.saude.gov.br/images/pdf/ 2017/setembro/06/2017_027.pdf

11. World Health Organization. Data From: Lower Doses of Yellow Fever Vaccine Could be Used in Emergencies. (2016). Available online at: https://www. who.int/en/news-room/detail/17-06-2016--lower-doses-of-yellow-fevervaccine-could-be-used-in-emergencies (accessed February 2019).

12. World Health Organization. WHO Secretariat Information Paper: Fractional Dose Yellow Fever Vaccine as a Dose-Sparing Option for Outbreak Response. (2016). Available online at: https://apps.who.int/iris/bitstream/handle/10665/ 246236/WHO-YF-SAGE-16.1-eng.pdf

13. Vannice K, Wilder-Smith A, Hombach J. Fractional-Dose yellow fever vaccination - advancing the evidence base. N Engl J Med. (2018) 379:603-5. doi: 10.1056/NEJMp1803433

14. Wilder-Smith A. Yellow fever vaccination: estimating coverage. Lancet Infect Dis. (2017) 17:1109-11. doi: 10.1016/S1473-3099(17)30494-2

15. Shearer FM, Moyes CL, Pigott DM, Brady OJ, Marinho F, Deshpande A, et al. Global yellow fever vaccination coverage from 1970 to 2016 : an adjusted retrospective analysis. Lancet Infect Dis. (2017) 17:1209-17. doi: 10.1016/S1473-3099(17)30419-X

16. Instituto de Tecnologia em Imunobiológicos/Bio-Manguinhos. Data From: Vacina de Febre Amarela de Bio Ajuda a Combater Epidemia em Angola. (2016). Available online at: https://www.bio.fiocruz.br/index.php/noticias/ 1218-vacina-de-febre-amarela-de-bio-ajuda-a-combater-surto-em-angola (accessed February 2019).

17. Roukens AH, Vossen AC, Bredenbeek PJ, van Dissel JT, Visser LG. Intradermally administered yellow fever vaccine at reduced dose induces a protective immune response: a randomized controlled non-inferiority trial. PLoS ONE. (2008) 3:e1993. doi: 10.1371/ journal.pone.0001993

18. Martins RM, Maia MdeLS, Farias RHG, Camacho LAB, Freire MS, Galler S, et al. 17DD yellow fever vaccine: a double blind, randomized clinical trial of immunogenicity and safety on a dose-response study. Hum Vacc Immunother. (2013) 9:879-88. doi: 10.4161/hv.22982

19. Campi-Azevedo AC, Estevam PdeA, Coelho-dos-Reis JG, PeruhypeMagalhães V, Villela-Rezende G, Quaresma PF, et al. Subdoses of 17DD yellow fever vaccine elicit equivalent virological/immunological kinetics timeline. BMC Infect Dis. (2014) 14:391. doi: 10.1186/1471-2334-14-391

20. de Menezes Martins R, Maia MLS, de Lima SMB, de Noronha TG, Xavier JR, Camacho LAB, et al. Duration of post-vaccination immunity to yellow fever in volunteers eight years after a dose-response study. Vaccine. (2018) 36:4112-7. doi: 10.1016/j.vaccine.2018.05.041

21. Roukens AHE, van Halem K, de Visser AW, Visser LG. Long-Term protection after fractional-dose yellow fever vaccination: follow-up study of a randomized, controlled, noninferiority trial. Ann Internal Med. (2018) 169:761-5. doi: 10.7326/M18-1529

22. Costa-Pereira C, Campi-Azevedo AC, Coelho-Dos-Reis JG, PeruhypeMagalhães V, Araújo MSS, do Vale Antonelli LR, et al. Multi-parameter approach to evaluate the timing of memory status after $17 \mathrm{DD}$ YF primary vaccination. PLoS Neglec Trop Dis. (2018) 12:e0006462. doi: 10.1371/journal.pntd.0006462

23. Ahuka-Mundeke S, Casey RM, Harris JB, Dixon MG, Nsele PM, Kizito GM, et al. Immunogenicity of fractional-dose vaccine during a yellow fever outbreak - preliminary report. N Engl J Med. (2018) 36:4112-7. doi: 10.1056/NEJMoa1710430

24. Gotuzzo E, Yactayo S, Cordova E. Review article: efficacy and duration of immunity after yellow fever vaccination: systematic review on the need for a booster every 10 years. Am J Trop Med Hygiene. (2013) 89:434-44. doi: 10.4269/ajtmh.13-0264

25. World Health Organization. Background paper on yellow fever vaccine. In: SAGE Working Group, editor. Vaccine Position Papers Weekly Epidemiological Record. Geneva: SAGE Working Group (2013). p. 269-84.

26. Reinhardt B, Jaspert R, Niedrig M, Kostner C, Lage-Stehr J. Development of viremia and humoral and cellular parameters of immune activation after vaccination with yellow fever virus strain 17D: a model of human flavivirus infection. J Med Virol. (1998) 56:159-67.

27. Poland JD, Calisher CH, Monath TP, Downs WG, Murphy K. Persistence of neutralizing antibody 30-35 years after immunization with $17 \mathrm{D}$ yellow fever vaccine. Bull World Health Organiz. (1981) 59:895-900.

28. Wieten RW, Jonker EF, van Leeuwen EM, Remmerswaal EB, Ten Berge IJ, de Visser AW, et al. A Single 17D Yellow fever vaccination provides lifelong immunity; characterization of yellow-fever-specific neutralizing antibody and t-cell responses after vaccination. PLOS ONE. (2016) 11:e0149871. doi: 10.1371/journal.pone.0149871

29. Akondy RS, Monson ND, Miller JD, Edupuganti S, Teuwen D, Wu H, et al. The yellow fever virus vaccine induces a broad and polyfunctional human memory CD8+ T cell response. J Immunol. (2009) 183:7919-30. doi: 10.4049/jimmunol.0803903

Conflict of Interest Statement: The authors declare that the research was conducted in the absence of any commercial or financial relationships that could be construed as a potential conflict of interest.

Copyright (C) 2019 Costa-Rocha, Campi-Azevedo, Peruhype-Magalhães, Coelho-dosReis, Fradico, Souza-Lopes, Reis, Freire, Costa-Pereira, Mambrini, Maia, Lima, Noronha, Xavier, Camacho, Albuquerque, Farias, Castro, Homma, Romano, Domingues, Martins, Teixeira-Carvalho and Martins-Filho. This is an open-access article distributed under the terms of the Creative Commons Attribution License (CC $B Y)$. The use, distribution or reproduction in other forums is permitted, provided the original author(s) and the copyright owner(s) are credited and that the original publication in this journal is cited, in accordance with accepted academic practice. No use, distribution or reproduction is permitted which does not comply with these terms. 